\section{Protective effect of curcumin in transgenic Drosophila melanogaster model of Parkinson's disease}

\author{
Yasir Hasan Siddique, 1 Gulshan Ara,2 \\ Smita Jyoti, 1 Mohammad Afzal2 \\ 1Drosophila Transgenic Laboratory and \\ 2Human Genetics and Toxicology \\ Laboratory, Section of Genetics, \\ Department of Zoology, Faculty of Life \\ Sciences, Aligarh Muslim University, \\ Uttar Pradesh, India
}

\section{Abstract}

Studies on model organisms have been found to be invaluable in clarifying the cellular and molecular basis of normal cellular processes and disease pathogenesis. Drosophila mutants and transgenes have provided a platform to understand the mechanisms associated with degenerative disease. Studies on the role of polyphenols in protecting against neurodegenerative diseases are limited. In the present study, the effect of curcumin at various doses was studied on the climbing ability of the transgenic Drosophila melanogaster that expresses normal human $\alpha$-synuclein in the neurons. A significant dose-dependent protection against loss of climbing ability was observed. The results suggest that curcumin can strongly improve the climbing ability of Parkinson's disease model flies and also supports the utility of this model in studying the symptoms of Parkinson's disease.

\section{Introduction}

Parkinson's disease (PD) is a common neurodegenerative syndrome characterized by the loss of dopaminergic neurons in the substantia nigra, formation of filamentous intraneuromal inclusions (Lewy bodies) and extra pyramidal movement disorders. ${ }^{1}$ PD is usually classified as a movement disorder, although it also gives rise to several non-motor types of symptoms, such as sensory deficits, cognitive difficulties or sleep problems. ${ }^{2}$ Two forms of human PD have been identified: the hereditary form, which accounts for less than 10\% of PD cases, and the idiopathic form, accounting for the remaining $90 \% .3,4$ At least $5 \%$ of people are now known to have forms of the disease that occur because of mutation of one of the several specific genes, ${ }^{5}$ such as alpha synuclein (SNCA), Ubiquitin Carboxy-terminal hydrolase
L1 (UCH-L1), parkin (PRKN), Leucine rich repeat kinase 2 (LRRK2), PTEN-induced putative kinase 1 (PINk1), DJ-1 and ATP BA2. The most extensively studied PD-related genes are SNCA and LRRK2.5-6 There are genetic models of PD based on $\alpha$-synuclein, primarily the transgenic overexpression of mutant or wildtype forms in mice or flies.7-11 Overexpression of either wild-type or mutant $\alpha$-synuclein in Drosophila leads to lewy body like synuclein containing inclusions and loss of dopaminergic neurons, as well as a behavioral abnormality that appears to be corrected by levodopa or dopaminergic agonist.1,12 Curcumin is the active ingredient of the turmeric plant (Curcumin longa). ${ }^{13}$ It has been shown to possess a number of pharmacological properties: antimutagenic, ${ }^{14}$ antigenotoxic, ${ }^{15,16}$ antitumor, ${ }^{14}$ anti-inflammatory, ${ }^{17}$ anti-ischemic, ${ }^{18}$ etc. We studied the effect of curcumin on the locomotor ability of Parkinson's disease model flies exhibiting $\alpha$-synuclein in the neurons.

\section{Materials and Methods}

\section{Drosophila stocks}

Transgenic fly lines that express wild-type human alpha synuclein under UAS control in neurons $\left(w I^{*}\right] ; P\{w /+m C]=U S A-H s a p / S N C A$. F\}" $5 B$ and GAL4 " $\left.w{ }^{*}\right] ; P\{w /+m C]=G A L 4$ elavL\}3 were obtained from Bloomington Drosophila stock center (Indiana University, Bloomington, IN, USA). When the males of UAS-Hsap/SNCA.F strains are crossed with the females of GAL4-elav.L (and vice-versa) the progeny will express the human $\alpha$-synuclein in their neurons. ${ }^{1}$

\section{Drosophila culture and crosses}

The flies were cultured on standard Drosophila food containing agar, corn meal, sugar and yeast at $25^{\circ} \mathrm{C}(24 \pm 1) .1^{19}$ Crosses were set up using 6 virgin females of UASHsap/SNCA.F5B who were mated to 3 GAL4elav males. The progeny will express the human $\alpha$-synuclein in the neurons and the flies were referred to as Parkinson's disease (PD) flies. First, the climbing assay was performed for the PD flies and the UASHsap/SNCA.F (control). The PD flies were exposed to different doses of curcumin mixed in the culture medium. Curcumin was added in the medium at final concentrations of 1 $\mu \mathrm{L} / \mathrm{mL}, 5 \mu \mathrm{L} / \mathrm{mL}$ and $10 \mu \mathrm{L} / \mathrm{mL}$. The UASHsap/SNCA.F were used as control. Vials of PD flies without curcumin were used as positive control.

\section{Drosophila climbing assay}

The climbing assay was performed as described by Pedleton et al. ${ }^{12}$ Ten flies were
Correspondence: Yasir Hasan Siddique, Drosophila Transgenic Laboratory, Section of Genetics, Department of Zoology, Faculty of Life Sciences, Aligarh Muslim University, Uttar Pradesh, India.

E-mail: yasir_hasansiddique@rediffmail.com

Key words: curcumin, Parkinson's disease, transgenic Drosophila, climbing ability.

Acknowledgments: we would like to thank the Chairman of the Department of Zoology for providing the laboratory facilities.

Received for publication: 18 August 2011. Accepted for publication: 2 December 2011.

This work is licensed under a Creative Commons Attribution NonCommercial 3.0 License (CC BYNC 3.0).

(C) Copyright Y.H. Siddique et al., 2012

Licensee PAGEPress, Italy

Alternative Medicine Studies 2012; 2:e3

doi:10.4081/ams.2012.e3

placed in empty glass vials $(10.5 \mathrm{~cm} \times 2.5 \mathrm{~cm})$. A horizontal line was drawn $8 \mathrm{~cm}$ above the bottom of the vial. After the flies had acclimated for 10 min at room temperature, both controls and treated groups were assayed at random for a total of 10 trials each. The procedure involved gently tapping the flies down to the bottom of the vials. The number of flies above the mark of the vial was counted after $10 \mathrm{sec}$ of climbing and repeated 10 times to get the mean number above the mark of the flies in each vial. These values were then averaged, and a group mean and standard error were obtained. The mean values of various fly groups were statistically compared using an unpaired group of Student's t-test. All behavioral studies were performed at $25^{\circ} \mathrm{C}$ under standard lighting conditions.

\section{Results and Discussion}

A time course evaluatin showed that the climbing response of control flies remained essentially unchanged over 21 days (Figure 1). From Day 9, however, the response of the PD flies was significantly lower than that of controls. Based on these results, standard duration of treatment was set at 21 days for the subsequent treatments with various doses of curcumin. The climbing assay was performed after 21 days of treatment. It was seen that 1.0, 5 and $10 \mu \mathrm{l} / \mathrm{ml}$ of curcumin significantly improved the deteriorating climbing ability of the PD flies (Figure 2).

The results of the present study show that the addition of curcumin to the culture medi- 
um significantly improved the climbing ability of the PD flies. A time-dependent loss of dopaminergic neurons in the dorsomedial group and the intracellular aggregates of $\alpha$ synuclein (Lewy bodies) were reported by Feany and Bender in the transgenic flies. These changes were followed by functional loss in climbing ability. ${ }^{1}$

PD is characterized by several abnormalities, including inflammation, mitochondrial dysfunction, iron accumulation, and oxidative stress.12 Pharmaceutical intervention in the molecular pathways of disease initiation and progression is one of the strategies currently being employed. Most research focuses on providing protection against the loss of dopaminergic neurons in the substantia nigra. The efficacy of the drug levodopa declines as PD progresses. Recently, there have been strategies aimed at preventing progressive cell death in the nigral dopamine neuron. ${ }^{11}$ Both wild-type and mutant $\alpha$-synuclein form amyloid fibrils resembling those seen in lewy body, as well as non-fibrillary oligomers termed protofibrills. The accumulation of $\alpha$-synuclein leads to toxicity and oxidative stress. ${ }^{20,21}$ It remains unclear whether misfolded proteins directly cause toxicity or damage cells via the formation of protein aggregates (Lewy body). ${ }^{11}$ However, in our present study, the treatment of PD flies with curcumin showed a protective effect and reduced the possible loss of climbing ability in PD flies as they got older. Curcumin is an antioxidant and a free radical scavenger. Its protective effect may be due to the reduction in oxidative stress, or due to the inhibition of the expression of the $\alpha$-synuclein, or by preventing the damage of dopaminergic neurons. It has been hypothesized that oxidative stress is linked to both the initiation and the progression of PD.

The present study was carried out using the Drosophila model of PD developed by Feany and Bender in 2000 that expresses human wild-type $\alpha$-synuclein in the neurons of the fly, with consequent locomotor dysfunction. ${ }^{1}$ The proteomic analysis of this panneural expression of human wild-type $\alpha$-synuclein in the transgenic flies showed a differential expression of proteins indicating a perturbation of molecular pathways involving metabolism and signaling. ${ }^{22}$ Gene expression changes for genes in these molecular pathways have been shown to be greatest in this model at the presymptomatic stage, when the potential for neuroprotection is greatest, thus validating this model for identifying potential targets for neuroprotective strategies. ${ }^{23}$ Similar results have been reported in the transgenic mice expressing human $\alpha$-synuclein in the neocortex, hippocampus, and substantia nigra. The mice showed progressive damage in the dopaminergic neurons and impaired rotorod performance. ${ }^{9}$

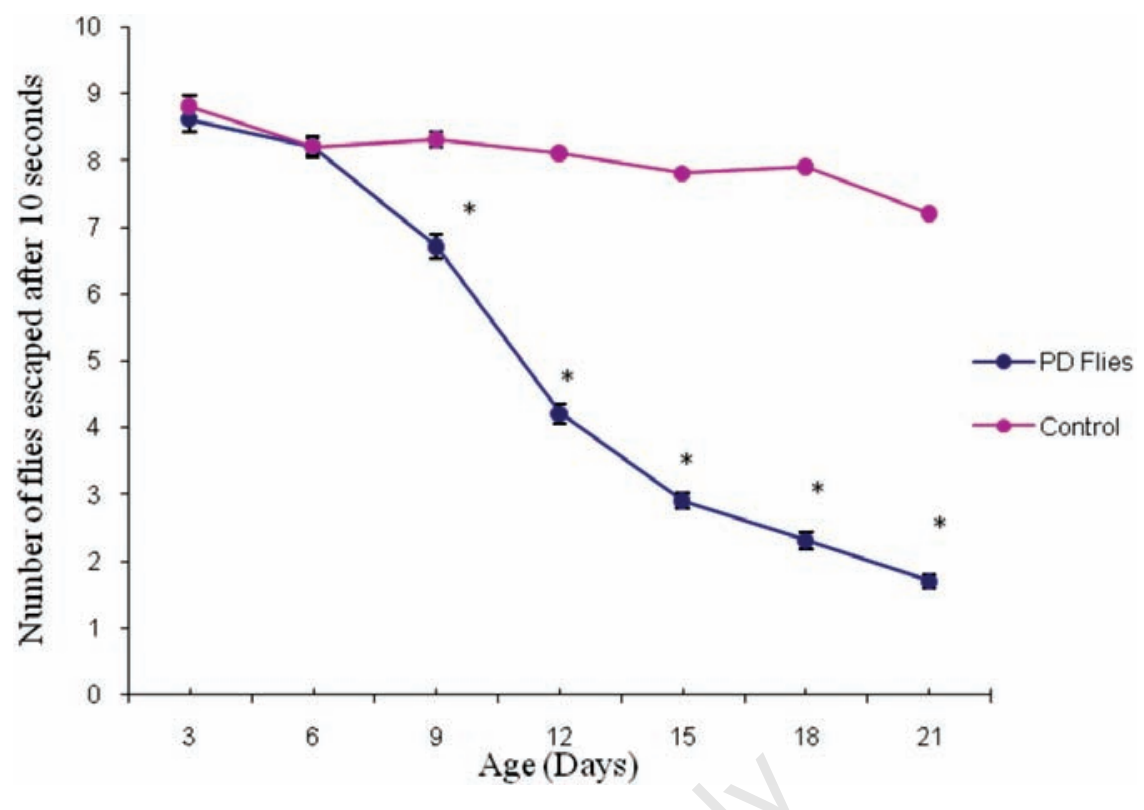

Figure 1. Climbing ability in Parkinson's disease flies and control over a period of 21 days. The values are the mean of 3 assays ( ${ }^{*}$ significant with respect to control $P<0.05$ ).

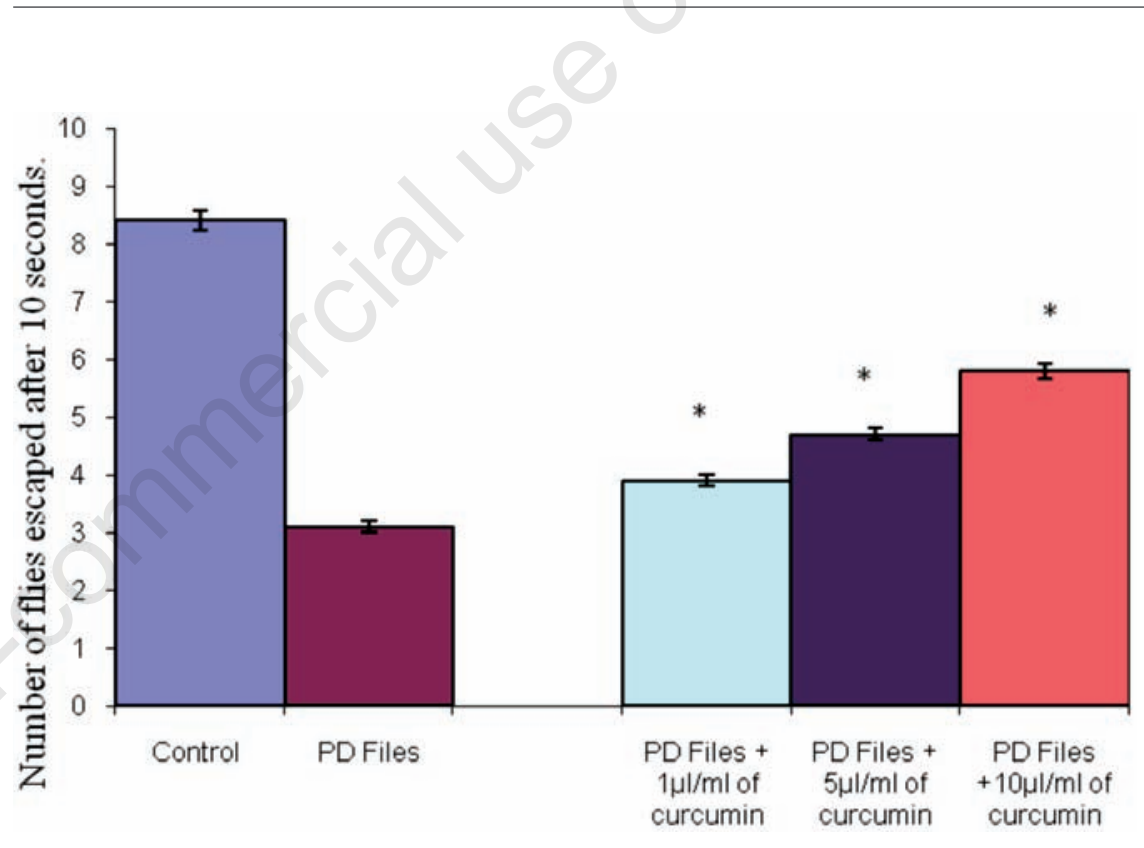

Figure 2. Effects of curcumin on fly climbing ability. The flies were treated with curcumin for 21 days and then assayed for climbing ability. Values are the mean of 3 assays ( ${ }^{*}$ significant with respect to $P D$ flies $P<0.05$ ).

The results of the present study suggest that the transgenic fly model mimics the motor impairments associated with PD and a climbing assay can be performed to determine whether or not a variety of compounds or drugs mixed in the fly culture medium prevent the progressive loss of climbing ability. 12

\section{References}

1. Feany MB, Bender WW. A drosophila model of Parkinson's disease. Nature 2000;404: 394-8.

2. Barnett-Cowan M, Dyde RT, Foxe SH, et al. 
Multisensory determinants of orientation perception in Parkinson's disease. Neurscience 2010;167:1138-50.

3. Drobysheva D, Ameel K, Welch B, et al. An optimized method for histological detection of dopaminergic neurons in Drosophila melanogaster. J Histochem Cytochem 2008;56:1049-63.

4. Nussbaum RL, Christopher EE. Alzheimer's disease and Parkinson's disease. N Engl J Med 2003;348:1356-64.

5. Davie CA. A review of Parkinson's disease. British Med Bull 2008;86:109-127.

6. Lesage S, Brice A. Parkinson's disease: from monogenic forms to genetic susceptibility factors. Hum Mol Genet 2009;18:4859.

7. Giasson B, Duda JE, Quinn SM, et al. Neuronal alpha-synucleinopathy with severe movement disorder in mice expressing A 53T human alpha synuclein. Neuron 2002;34:521-33.

8. Lee HJ, Shin SY, Choi C, et al. Formation and removal of alpha-synuclein aggregates in cells exposed to mitochondrial inhibitors. J Biol Chem 2002;277:5411-7.

9. Masliah E, Rockenstein E, Veinbergs I, et al. Dopaninergic loss and inclusion body formation in alpha-synuclein mice: implication for neuro-generative disorders Science 2000;287:1265-9.

10. Vander putten $\mathrm{H}$, Wiederhold $\mathrm{KH}$, Probst A, et al. Neuropathology in mice expressing human alpha synuclein. J Neurosci 2000; 20:6021-9.

11. Dauer W, Przedborski S. Parkinson's disease: Mechanisms and models. Neuron 2003;39:889-909.

12. Pendleton RG, Parvez F, Sayed M, et al. Effects of pharmacological agents upon a transgenic model of Parkinson's disease in Drosophila melanogaster. J Pharmacol Exp Ther 2002;300:91-6.

13. Chattopadhyay J, Biswas K, Bandy-opadhyay U, et al. Tumeric and curcumin: biological actions and medicinal applications. Curr Sci 2004;87:44-53.

14. Shishu, Kaur IP. Antimutagenicity of curcumin and related compounds against genotoxic heterocyclic amines from cooked food: The structural requirement. Food Chem 2008;111:573-9.

15. Siddique YH, Ara G, Beg T, et al. Protective effect of curcumin against the genotoxic damage induced by tinidazole in culture human lymphocytes. Acta Pharm Sci 2010;52:23-30.

16. Siddique YH, Ara G, Beg T, et al. Protective effect of curcumin against chlormadinone acetate induced genotoxic damage in cultured human peripheral blood lymphocytes. Pharmacology online 2010;3:644-50.

17. Srivastava KC, Bordia A, Verma SK. Curcumin, a major component of the food spice turmeric (curcuma longa), inhibits aggregation and alters eicosanoid metabolism in human blood platelets. Prostaglandins Leuk Ess Fatty Acids 1995;52:223-7.

18. Shukla PK, Khanna Vk, Ali MM, et al. Antiischemic effect of curcumin in rat brain. Neurochem Res 2008;33:1036-43.

19. Siddique YH, Ara G, Beg T, et al. Protective role of Plumbago zeylanica extract against toxic effects of ethinylestradiol in the third instar larvae of transgenic Drosophila melanogaster (hsp70-lacZ)Bg9 and culture human peripheral blood lymphocytes. Alt Med Studies 2011;e7.

20. Conway KA, Harper JD, Lansbury PT Accelerated in vitro fibril formation by a mutant alpha synuclein linked to early onset Parkinson disease. Nat Med 1988; 4:1318-20.

21. Giasson BI, Ury UK, Trojanowski JQ, et al. Mutant and wild type human alpha synuclein assemble into elongated filaments with distinct morphologies in vitro. $\mathrm{J}$ Biol Chem 1999;274:7619-22.

22. Xun Z, Kaufman TC, Clemmer DE Proteome response to the panneural expression of human wild type apha synuclein: A Drosophila model of Parkinson's disease. J Proteome Res 2008; 7:3911-21.

23. Scherzer CR, Jensen RV, Gullans SR, et al. Gene expression changes presage neurodegeneration in a Drosophila model of Parkinson's disease. Hum Mol Genet 2003;12:2457-66. 